\title{
Algorithm for automated tuning of a quantum dot into the single-electron regime
}

\author{
M. Lapointe-Major $\odot,{ }^{1, *}$ O. Germain $\odot,{ }^{2}$ J. Camirand Lemyre, ${ }^{1}$ D. Lachance-Quirion, ${ }^{1}$ S. Rochette,${ }^{1}$ \\ F. Camirand Lemyre, ${ }^{2}$ and M. Pioro-Ladrière $\oplus^{1}$ \\ ${ }^{1}$ Institut quantique and Département de Physique, Université de Sherbrooke, Sherbrooke, Québec, Canada J1K $2 R 1$ \\ ${ }^{2}$ Département de Mathématiques, Université de Sherbrooke, Sherbrooke, Québec, Canada J1K 2RI
}

(Received 14 January 2020; revised 17 April 2020; accepted 27 May 2020; published 3 August 2020)

\begin{abstract}
We report an algorithm designed to perform computer-automated tuning of a single quantum dot with a charge sensor. The algorithm performs an adaptive measurement sequence of subsized stability diagrams until the single-electron regime is identified and reached. For each measurement, the signal processing module removes the physical background of the charge sensor to generate a binary image of charge transitions. Then, the image analysis module identifies the position and number of lines using two line detection schemes that are robust to noise and missing data.
\end{abstract}

DOI: 10.1103/PhysRevB.102.085301

\section{INTRODUCTION}

Spin qubits in quantum dots are among the frontrunner architectures for the implementation of a small-scale quantum computer [1,2] due to their high potential for scalability [3-6]. Indeed, progress towards devices with multiple quantum dots was recently demonstrated $[7,8]$. However, as the number of quantum dots increases, the brute-force approach of manually adjusting several gate voltages per quantum dot to reach the qubit regime has become impractical. To date, software has been developed to address this issue by automatizing tedious parts of this process for double dots using image analysis or machine learning tools to adjust the interdot tunnel coupling [9], detect triple points in stability diagrams [10,11], and perform state recognition [12-15].

In this paper, we report an algorithm designed to perform automated tuning of a single quantum dot tunnel coupled to a reservoir of electrons using only charge sensing. This has been recognized as challenging for the following reasons: (i) Tuning a single dot requires line detection, which proves to be less robust than the detection of triple points [10]. (ii) The number of transitions in a measurement is a priori unknown. (iii) The detection of transition lines with possible curvature and in the presence of noise and missing data points is computationally expensive [16]. (iv) The charge sensor couples to all charges at proximity, thus measuring several unwanted features, giving rise to a physical background in the resulting signal.

Our algorithm achieves all this by performing an iterative sequence of measurements, analysis, and state detection until the single-electron regime is reached [Fig. 1(a)] and requires

\footnotetext{
*maxime.lapointe-major@usherbrooke.ca
}

Published by the American Physical Society under the terms of the Creative Commons Attribution 4.0 International license. Further distribution of this work must maintain attribution to the author(s) and the published article's title, journal citation, and DOI. only the charge sensor to be precalibrated with minimal user inputs. Given a measurement, the signal processing module removes the physical background of the charge sensor to generate a map of detected charge transitions, and the image analysis module reconstructs the transition lines from that map. The latter was implemented using two different approaches, namely, a modified Hough transform [17] and the EDLines algorithm [18], each of which have different advantages regarding computation time and detection of curved transitions. Finally, a sequence of measurements that allows the algorithm to reach the single-electron regime is introduced. This sequence is based on a heuristic that aims to find the quantum dot regime, empty it, and then add one electron.

\section{SIGNAL PROCESSING}

The signal processing is designed to remove the physical background of the charge sensor and identify voltages where a charge transition occurs in a measurement.

In our setup, a single quantum dot is tunnel coupled to a reservoir of electrons in the split accumulation gate geometry [6] [Fig. 1(b)]. The quantum dot is capacitively coupled to a single-electron transistor (SET), used for charge sensing. The current through the SET $I_{\mathrm{SET}}$ is measured with a 1MHz-bandwidth cryogenic amplifier [19]. In that setup, ISET is monitored as a function of gate voltages [Fig. 2(a)] to detect transitions in the quantum dot electron occupancy. We model the current through the SET following

$$
\begin{aligned}
I_{\mathrm{SET}} & =A\left(V_{\mathrm{g}}\right) \sin \left[\Omega\left(V_{\mathrm{g}}\right)\right]+B\left(V_{\mathrm{g}}\right), \\
\Omega\left(V_{\mathrm{g}}\right) & =\omega\left(V_{\mathrm{g}}\right) V_{\mathrm{g}}+\phi(N) .
\end{aligned}
$$

The current contains a zero- and low-frequency background term $B\left(V_{\mathrm{g}}\right)$ and an oscillating term with a voltagedependent amplitude $A\left(V_{\mathrm{g}}\right)$ and argument $\Omega\left(V_{\mathrm{g}}\right)$. The frequency $\omega\left(V_{\mathrm{g}}\right)$ depends on gate voltage in two ways. First, it can be slowly modulated by gate voltage since the charging energy of the SET can vary over large ranges of gate voltage [20]. Here, this effect is mitigated by performing 
(a)

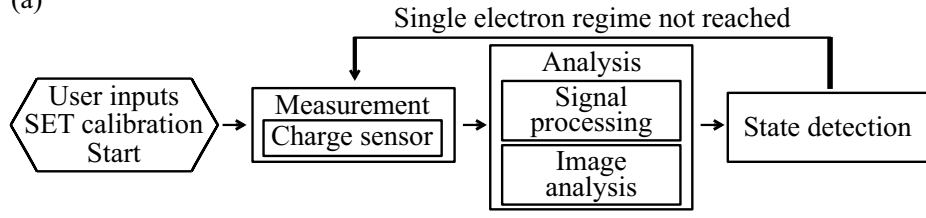

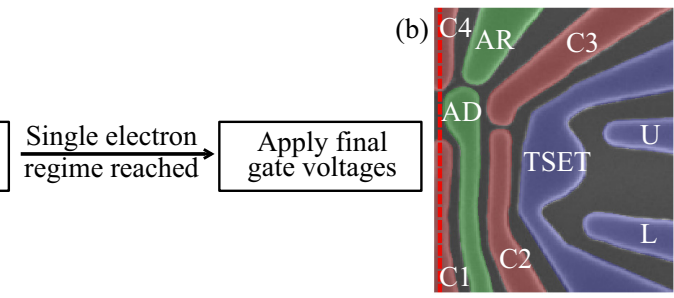

FIG. 1. (a) Flow chart of the algorithm. A subsized stability diagram is first measured by the charge sensor. This measurement goes through the signal processing module, which removes the physical background of the charge sensor and generates a binary image of the detected transitions. The image analysis module then identifies transition lines in that binary image. If the single-electron regime is reached, the algorithm sets gate voltages appropriately and otherwise loops again in the measurement and analysis sequence. (b) False-color scanning electron micrograph of a device nominally identical to the one used for the measurements. A mirror structure, on the left side of the red dashed line, not shown for clarity, includes gates $\mathrm{AD}^{\prime}, \mathrm{AR}^{\prime}, \mathrm{C2}^{\prime}, \mathrm{C}^{\prime}, \mathrm{TSET}^{\prime}, \mathrm{U}^{\prime}$, and $\mathrm{L}^{\prime}$. TSET, $\mathrm{U}$, and L (blue) are used to form a single-electron transistor (SET) used for charge sensing and require pretuning from the user. Confinement gates C1, C2, C3, and C4 (red) are roughly set to a predefined operating point. AD and AR (green) are the dot enhancement gate and the reservoir enhancement gate, respectively.

measurements over ranges such that this effect can be neglected. Second, the term $\phi(N)$, which models jumps that occur in $I_{\text {SET }}$ when an electron is added in the quantum dot, can be included in $\omega\left(V_{\mathrm{g}}\right)$. Therefore, the frequency $\omega\left(V_{\mathrm{g}}\right)$ remains constant except when a voltage-dependent charge transition $\Delta N\left(V_{\mathrm{g}}\right)$ occurs. The argument of the sine, $\Omega\left(V_{\mathrm{g}}\right)$, can then be rewritten as $\Omega\left(V_{\mathrm{g}}\right)=\omega\left(\Delta N\left(V_{\mathrm{g}}\right)\right) V_{\mathrm{g}}$. For the purpose of charge detection, the only components of interest in $I_{\mathrm{SET}}$ are the transitions in the electron occupancy of the quantum dot $\Delta N$.

In the signal processing module, the goal is to identify gate voltages for which a charge transition $\Delta N$ occurs. This is achieved by removing all the other components in the signal. First, a fifth-order Butterworth high-pass filter [21] is applied to the measured signal to remove the background term $B\left(V_{\mathrm{g}}\right)$. The cutoff frequency for the filter is extracted by fitting a Lorentzian to the Fourier transform of the signal [Fig. 2(b)]. Next, the instantaneous frequency of the oscillations is extracted using a Hilbert transform of the filtered signal [Fig. 2(a)]. The instantaneous frequency shows negative jumps at gate voltages where a charge transition occurs. These jumps are identified by computing an adaptive threshold $T$ taking into account the average $\bar{\omega}$ and standard deviation
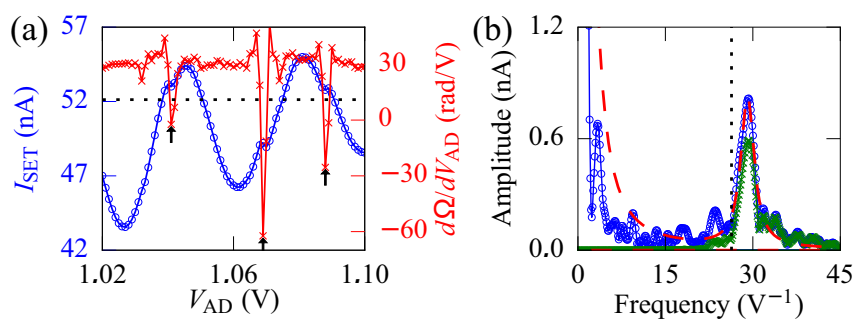

FIG. 2. (a) Typical $I_{\mathrm{SET}}$ trace (blue line with circles) and instantaneous frequency of the $I_{\mathrm{SET}}$ oscillations (red line with crosses) as a function of the dot gate (AD) voltage. Arrows indicate detected transitions, and the dotted line shows the threshold computed for this frequency distribution. (b) Fourier transform of the data shown in (a) (blue line with circles), the Lorentzian fit (red dashed line) used to extract the cutoff frequency for the high-pass filter (vertical dotted line), and the Fourier transform of the filtered signal (green line with crosses). $\sigma_{\omega}$ of the distribution of frequencies. Any point below the threshold is then identified as a charge transition. Using a severe threshold $\left(T>\bar{\omega}-3.5 \sigma_{\omega}\right)$ yields several false-negative results, while a loose threshold $\left(T<\bar{\omega}-2 \sigma_{\omega}\right)$ yields several false-positive results. We find the image analysis module to perform best using $T=\bar{\omega}-3 \sigma_{\omega}$ for the threshold.

Typical measurements used to identify charge transitions are two-dimensional stability diagrams where $V_{\mathrm{AD}}$ is swept and $V_{\mathrm{AR}}$ is stepped after every $V_{\mathrm{AD}}$ sweep [Fig. 3(a)].
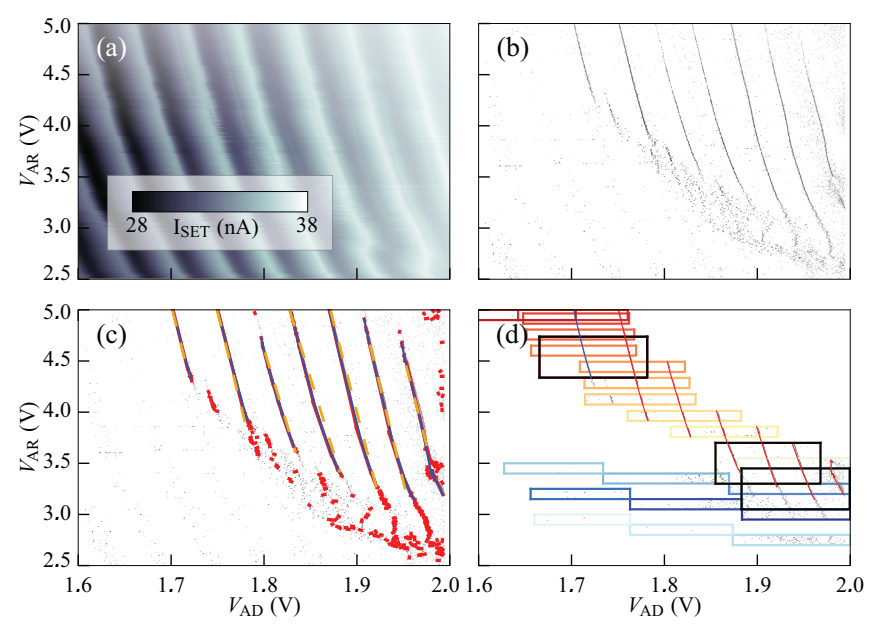

FIG. 3. (a) Experimentally measured stability diagram on a split accumulation gate quantum dot. $\mathrm{AD}$ is the dot gate with a $0.5-\mathrm{mV}$ resolution (801 pixels), and AR is the reservoir gate with a $2.5-\mathrm{mV}$ resolution (1001 pixels). (b) Binary image of the transition points detected by the signal processing module for the stability diagram shown in (a). (c) Transition lines detected by the image analysis module with the EDLines algorithm (yellow dashed lines) or the modified Hough transform before (red segments) and after (blue lines) the line reconstruction protocol. Red segments without a matching blue line are identified as false-positive transition lines and discarded. (d) Measurement sequence used by the algorithm to reach the single-electron regime. The sequence of measurement follows the color spectrum with the first sampled subdiagram in dark blue and the last sampled subdiagram in dark red. Larger subdiagrams sampled to confirm transitions are in black. Transition lines detected by the image analysis after the tuning process is completed are shown in red, and the first charge transition is in blue. 
Two-dimensional measurements provide many advantages to the algorithm: (i) they improve robustness to noise when falsepositive transitions are detected; (ii) they allow the algorithm to extrapolate information in regions where the charge sensor sensitivity is reduced, and (iii) they provide information about the ratio of the lever arms of the two gates.

Even though the measured stability diagrams are twodimensional, the signal processing procedure is kept onedimensional to circumvent the detection of telegraphic noise as a transition line by the image analysis module. The result of the signal processing module applied to a large stability diagram is a binary map of detected transitions [Fig. 3(b)].

Compared to other existing solutions to remove the physical background from charge sensor measurements [22,23], this approach does not require additional hardware and requires only postmeasurement processing.

\section{IMAGE ANALYSIS}

The goal of the image analysis module is to identify transition lines from the binary image output of the signal processing module. The main challenge of this step arises from the charge sensor not providing any way to label the detected transition points. This means the algorithm must identify (i) to which transition line each of the transition points belongs, (ii) the number of transition lines in a given measurement, and (iii) their position. Measured transitions must be detected amid device and signal processing noise, missing data points, and curvature of the transitions. This has led to two implementations for this module using either a modified Hough transform [17] or EDLines [18] for the efficient detection of curved lines or fast computation time, respectively.

The modified Hough transform is implemented in a divideand-conquer approach, where segments are recursively split into smaller, more manageable ones before being either discarded or reconstructed into charge transition lines. First, the algorithm generates clusters of neighboring pixels using a linkage algorithm based on connectivity of the pixels. Each cluster is defined to be a distance of at least one pixel from its neighboring clusters. For each of the clusters generated this way, a modified Hough transform is computed which estimates the best segment describing the cluster along with the covariance matrix as described in Ref. [17]. Here, a correction is applied to the covariance matrix to account for the width of the transition lines in the stability diagrams, which comes from the statistical nature of the tunneling events and the tunnel coupling between the dot and the reservoir. Following Ref. [24], clusters are recursively split to break down curved clusters into smaller pieces that can be appropriately approximated by a linear segment [red segments in Fig. 3(c)], and transition lines are reconstructed based on collinearity and proximity of the end point criteria [blue segments in Fig. 3(c)].

The second implementation uses the EDLines algorithm in a top-down approach. The EDLines algorithm identifies anchor points to generate segments with very few false positives. Here, the width of the charge transitions is typically more than one pixel. This leads to the detection of several segments for each transition by the EDLines algorithm, each shifted by one pixel. Therefore, doublons must be discarded. This is achieved using the parallelism criteria in Ref. [24]. Next, segments are regrouped and identified as transition lines based on the same collinearity and proximity of the end point criteria used for the modified Hough transform.

Both implementations of the image analysis module are robust to missing data points (false negatives) and noise (false positives) [17,18]. This has been verified on a number of stability diagrams acquired on two different devices and is pictured in Fig. 3(c), where line reconstruction succeeds despite noise surrounding the transitions. On these devices, falsepositive and false-negative instances are always in sufficiently low occurrence to guarantee the success of our algorithm. Missing data can occur for a variety of reasons: (i) they can be due to a loss of sensitivity of the SET, which can be caused by the modulation of the tunnel barriers in the SET due to interface irregularities [25]; (ii) they can be introduced by the signal processing module when using a severe threshold $T$, or (iii) they can be introduced by the measurement sequence [Fig. 3(d)]. Typical sources of noise in stability diagrams are charge noise, current noise in the SET, telegraphic noise and false-positive transition points added by the signal processing module. The similar performances of the two algorithms in the presence of typical noise is explained by the fact that EDLines identifies very few false-positive transition lines by design [18], while the line reconstruction of the modified Hough transform is very efficient at identifying and discarding them [red segments in Fig. 3(c)].

The modified Hough transform implementation is expected to be more efficient at detecting charge transitions with large curvature because of the initial construction of transition lines, which regroups all transition points into a cluster independent of the curve before splitting and reconstructing it in its divideand-conquer scheme. These transitions with large curvature would not be detected by the EDLines algorithm due to its validation method [18]. This has been qualitatively observed in some experimental data sets. In the presence of incidental quantum dots caused by defects, charge transition lines of the defect can cross with the quantum dot's charge transition lines at various points within a stability diagram, causing $X$-shaped clusters at these points. The modified Hough transform is expected to perform poorly in these conditions and sometimes regroup segments belonging to different charge transitions within the same line. Detection of the line crossings and division of the $\mathrm{X}$-shaped clusters at these line crossings would allow proper line reconstruction by the modified Hough transform. EDLines is expected to reconstruct lines properly [18] in these conditions. Finally, both implementations were successful at reconstructing lines even in the presence of telegraphic noise, provided the jumps are smaller than half the distance between charge transitions.

For the stability diagram in Fig. 3(c), the image analysis requires $45 \mathrm{~s}$ to identify the blue transition lines using the modified Hough transform implementation and requires $1.5 \mathrm{~s}$ to identify the yellow dashed transition lines using the EDLines implementation on an Intel Xeon CPU E3-1245 v5 running at $3.50 \mathrm{GHz}$. This speedup is consistent with results obtained on other experimental stability diagrams. 


\section{MEASUREMENT SEQUENCE}

Prior to starting the algorithm, the user specifies the starting voltages for $V_{\mathrm{AD}}$ and $V_{\mathrm{AR}}$, the maximum and minimum voltages allowed by the algorithm and the voltage resolution to be used throughout the tuning procedure. The voltage resolution is kept fixed through all measurements to avoid pixel connectivity issues that would arise due to the changing pixel size and voltage grid. Finally, the user is required to manually calibrate the charge sensor in a suitable operating regime.

A heuristic algorithm (see the Supplemental Material [26]) is now applied to find the last charge transition following the flowchart in Fig. 1. The goal of the measurement sequence is to acquire enough information about the quantum dot to identify the single-electron regime with the fewest measurements possible. This heuristic mimics a typical tuning protocol, where the dot is first formed and emptied until the last transition is found based on subsized stability diagrams measured using only two accumulation gates $\left(V_{\mathrm{AD}}\right.$ and $\left.V_{\mathrm{AR}}\right)$. In the accumulation split gate geometry, the accumulation gates of the quantum dot and of its reservoir are selected since they offer full control over physical quantities meaningful to the algorithm [6], while confinement gates are roughly set to a predefined operating point and require no fine tuning. This could be extended to more complex gate architectures using the concept of virtual gates $[11,27,28]$.

When the tuning routine is initiated, a subdiagram is first measured in the user-specified scan range. If no transition is detected, the algorithm performs a diagonal series of measurements. When a measurement would fall beyond the voltage restriction, a new diagonal series with a different initial $V_{\mathrm{AR}}$ is initiated, alternating between higher and lower $V_{\mathrm{AR}}$. This is done recursively until a charge transition is detected. To verify the detected line truly belongs to a transition and is not an artifact of the signal processing or due to experimental noise, a larger subdiagram is measured centered on the leftmost detected line. If the transition is confirmed, the program then follows the transition by increasing $V_{\mathrm{AR}}$ until it disappears due to broadening. Then, lower $V_{\mathrm{AD}}$ measurements are performed until the next transition is found. This is done recursively until no more transitions are found, meaning the quantum dot is empty. Finally, the program analyzes the ensemble of all completed measurements and identifies the first charge transition [Fig. 3(d)]. A larger subdiagram is then measured on this transition to confirm it, and gate voltages are adjusted to add one electron back into the quantum dot. When following or confirming a transition, measurements that would fall beyond the user-specified voltage limitations are shifted within the restrictions. This measurement sequence was tested by sampling subdiagrams from large experimental stability diagrams taken on two different devices. The algorithm proved successful in all tested instances, even in the presence of telegraphic noise. In the presence of defects, the algorithm is expected to reach the single-electron regime, but the electron might be added in the defect instead of the quantum dot. Future work includes the discrimination of defects' charge transitions by taking into account the slopes of the transitions.

The measurement sequence takes advantages of the robustness to missing data of the image analysis module by leaving blank spaces between measurements [Fig. 3(d)], which allows us to reduce measurement time. Segments from different measurements are regrouped only at the end of the sequence. This reduces the computational cost as the image analysis module is called only once per measurement with one extra call for the sum of all measurements.

The scan range of each subdiagram is limited by the signal processing module. The extraction of the cutoff frequency for the high-pass filter and the Hilbert transform both yield significantly better results when a full cycle of the SET background oscillations is acquired. Therefore, prior to the measurement of a subdiagram, a preliminary sweep of $V_{\mathrm{AD}}$ is performed to estimate the background frequency. The width of the stability diagram is then determined to include at least a full cycle of the oscillations. The height of the stability diagram is arbitrarily chosen to be 40 pixels, which yields a good enough transition length for the line reconstruction given a typical transition width, which is three or four pixels wide when using a gate voltage resolution of approximately $1 \mathrm{mV}$.

\section{CONCLUSION}

In summary, we have developed an algorithm designed to tune a single quantum dot to the single-electron regime. We have shown a protocol to remove the physical background from charge sensor measurements that can loosen the requirements for additional hardware and feedback loops in SETbased charge detection. Furthermore, we have developed and compared two image analysis algorithms to identify charge transitions. While EDLines is at least ten times faster, the modified Hough transform approach is believed to present additional robustness to line curvature. We envision that combined with recently demonstrated identification of triple points $[10,11]$ and the concept of virtual gates $[11,27,28]$, our algorithm will provide additional tools for automated initialization and control routines of quantum dots.

\section{ACKNOWLEDGMENTS}

We thank our collaborators at Sandia National Laboratories for providing the samples used for the development of our algorithm. We recognize helpful conversations with J. K. Gamble (Sandia National Laboratories) and C. Lupien about the computer-assisted visual approach. We acknowledge useful comments and discussions with G. Brookes, J. O. Simoneau, and R. H. Foote. This work was supported by the Natural Sciences and Engineering Research Council of Canada (NSERC) and the Canada Foundation for Innovation (CFI). This research was undertaken thanks in part to funding from the Canada First Research Excellence Fund. All developed code and additional data are available upon request to M.-L.-M. 
[1] D. Loss and D. P. DiVincenzo, Phys. Rev. A 57, 120 (1998).

[2] F. A. Zwanenburg, A. S. Dzurak, A. Morello, M. Y. Simmons, L. C. L. Hollenberg, G. Klimeck, S. Rogge, S. N. Coppersmith, and M. A. Eriksson, Rev. Mod. Phys. 85, 961 (2013).

[3] L. M. K. Vandersypen, H. Bluhm, J. S. Clarke, A. S. Dzurak, R. Ishihara, A. Morello, D. J. Reilly, L. R. Schreiber, and M. Veldhorst, npj Quantum Inf. 3, 34 (2017).

[4] P. Harvey-Collard, N. T. Jacobson, M. Rudolph, J. Dominguez, G. A. Ten Eyck, J. R. Wendt, T. Pluym, J. K. Gamble, M. P. Lilly, M. Pioro-Ladrière, and M. S. Carroll, Nat. Commun. 8, 1029 (2017).

[5] T. M. Lu, J. K. Gamble, R. P. Muller, E. Nielsen, D. Bethke, G. A. Ten Eyck, T. Pluym, J. R. Wendt, J. Dominguez, M. P. Lilly, M. S. Carroll, and M. C. Wanke, Appl. Phys. Lett. 109, 093102 (2016).

[6] S. Rochette, M. Rudolph, A.-M. Roy, M. J. Curry, G. A. T. Eyck, R. P. Manginell, J. R. Wendt, T. Pluym, S. M. Carr, D. R. Ward, M. P. Lilly, M. S. Carroll, and M. Pioro-Ladrière, Appl. Phys. Lett. 114, 083101 (2019).

[7] D. M. Zajac, T. M. Hazard, X. Mi, E. Nielsen, and J. R. Petta, Phys. Rev. Appl. 6, 054013 (2016).

[8] A. R. Mills, D. M. Zajac, M. J. Gullans, F. J. Schupp, T. M. Hazard, and J. R. Petta, Nat. Commun. 10, 1063 (2019).

[9] C. J. van Diepen, P. T. Eendebak, B. T. Buijtendorp, U. Mukhopadhyay, T. Fujita, C. Reichl, W. Wegscheider, and L. M. K. Vandersypen, Appl. Phys. Lett. 113, 033101 (2018).

[10] T. A. Baart, P. T. Eendebak, C. Reichl, W. Wegscheider, and L. M. K. Vandersypen, Appl. Phys. Lett. 108, 213104 (2016).

[11] A. R. Mills, M. M. Feldman, C. Monical, P. J. Lewis, K. W. Larson, A. M. Mounce, and J. R. Petta, Appl. Phys. Lett. 115, 113501 (2019).

[12] S. S. Kalantre, J. P. Zwolak, S. Ragole, X. Wu, N. M. Zimmerman, M. D. Stewart, and J. M. Taylor, npj Quantum Inf. 5, 6 (2019).

[13] J. P. Zwolak, T. McJunkin, S. S. Kalantre, J. P. Dodson, E. R. MacQuarrie, D. E. Savage, M. G. Lagally, S. N. Coppersmith, M. A. Eriksson, and J. M. Taylor, Phys. Rev. Appl. 13, 034075 (2020).
[14] J. Darulová, S. J. Pauka, N. Wiebe, K. W. Chan, G. C. Gardener, M. J. Manfra, M. C. Cassidy, and M. Troyer, Phys. Rev. Appl. 13, 054005 (2020).

[15] R. Durrer, B. Kratochwil, J. V. Koski, A. J. Landig, C. Reichl, W. Wegscheider, T. Ihn, and E. Greplova, Phys. Rev. Appl. 13, 054019 (2020).

[16] V. Leavers, CVGIP: Image Understanding 58, 250 (1993).

[17] L. A. Fernandes and M. M. Oliveira, Pattern Recognit. 41, 299 (2008).

[18] C. Akinlar and C. Topal, Pattern Recognit. Lett. 32, 1633 (2011).

[19] M. J. Curry, M. Rudolph, T. D. England, A. M. Mounce, R. M. Jock, C. Bureau-Oxton, P. Harvey-Collard, P. A. Sharma, J. M. Anderson, D. M. Campbell, J. R. Wendt, D. R. Ward, S. M. Carr, M. P. Lilly, and M. S. Carroll, Scientific Reports 9, 16976 (2019).

[20] B. Meurer, D. Heitmann, and K. Ploog, Phys. Rev. Lett. 68, 1371 (1992).

[21] H. Zumbahlen, Linear Circuit Design Handbook (Newnes, 2008).

[22] C. H. Yang, W. H. Lim, F. A. Zwanenburg, and A. S. Dzurak, AIP Adv. 1, 042111 (2011).

[23] C. Barthel, M. Kjærgaard, J. Medford, M. Stopa, C. M. Marcus, M. P. Hanson, and A. C. Gossard, Phys. Rev. B 81, 161308(R) (2010).

[24] D. G. Lowe, Artif. Intell. 31, 355 (1987).

[25] E. P. Nordberg, G. A. Ten Eyck, H. L. Stalford, R. P. Muller, R. W. Young, K. Eng, L. A. Tracy, K. D. Childs, J. R. Wendt, R. K. Grubbs, J. Stevens, M. P. Lilly, M. A. Eriksson, and M. S. Carroll, Phys. Rev. B 80, 115331 (2009).

[26] See Supplemental Material at http://link.aps.org/supplemental/ 10.1103/PhysRevB.102.085301 for pseudocode summarizing key steps of the algorithm .

[27] T. Hensgens, T. Fujita, L. Janssen, X. Li, C. Van Diepen, C. Reichl, W. Wegscheider, S. D. Sarma, and L. M. Vandersypen, Nature (London) 548, 70 (2017).

[28] C. Volk, A. Zwerver, U. Mukhopadhyay, P. Eendebak, C. Van Diepen, J. Dehollain, T. Hensgens, T. Fujita, C. Reichl, W. Wegscheider et al., npj Quantum Inf. 5, 29 (2019). 